\title{
Aluminum Nano-polycrystalline Substance with Ferromagnetics and Application to High-Frequency Core Inductor
}

\author{
Mitsuru Inada*, Yukio Iida, Taku Saiki, Shinichirou Masuda \\ Faculty of Engineering Science, Kansai University, Osaka, Japan
}

Email address:

inada@kansai-u.ac.jp (M. Inada)

${ }^{*}$ Corresponding author

To cite this article:

Mitsuru Inada, Yukio Iida, Taku Saiki, Shinichirou Masuda. Aluminum Nano-polycrystalline Substance with Ferromagnetics and Application to High-Frequency Core Inductor. Journal of Electrical and Electronic Engineering. Vol. 5, No. 3, 2017, pp. 98-103.

doi: $10.11648 /$ j.jeee. 20170503.13

Received: April 11, 2017; Accepted: April 22, 2017; Published: June 21, 2017

\begin{abstract}
It has showed in experiments that aluminum nano-polycrystalline substances are ferromagnetic. A magnetic hysteresis curve measured by SQUID suggested the ferromagnetism. Al bulk is normally thought to be non-magnetic body. Authors also fabricated core inductors using $\mathrm{Al}$ nano-polycrystalline substances and measured the inductances of cored inductors with frequency dependence. Al nano-polycrystalline substance was fabricated by sintering $\mathrm{Al}$ nanopaste with $\mathrm{Al}$ nanoparticles. Al nanoparticles were prepared by using laser ablation in liquid. The structures and components of the sintered Al nano-polycrystalline substances were analyzed by SEM and EDX. It has been shown that inductor using $\mathrm{Al}$ nano-polycrystalline substance with low volume resistivity, which is the same order of metal, works at low frequencies below $500 \mathrm{~Hz}$, while inductor using substance with high volume resistivity works at high frequency of $5 \mathrm{MHz}$. Our analysis of $\mathrm{Al}$ nano-polycrystalline substance with high volume resistivity revealed relative permittivity of 7 at frequency of $1 \mathrm{MHz}$. It has been expected that these inductors to work at frequency of a few $\mathrm{GHz}$ because the magnetic resonance frequency of the $\mathrm{Al}$ nano-polycrystalline substances were evaluated to be $5.6 \mathrm{GHz}$ and the high volume resistivity results in suppressing the eddy currents.
\end{abstract}

Keywords: Al Nano-polycrystalline, Laser Ablation in Liquids, Core Inductors, High Frequency, Ferro-magnetic, SQUID

\section{Introduction}

Electronic devices are operating at increasingly higher frequencies, and the development of a cored inductor working at the frequency of few $\mathrm{GHz}$ regime is now required [1-8]. A granular tin film produced by the CVD process [4] and a sheet with small particles diffused in resins has been developed for use as inductor cores [5-7], which are magnetic materials working at a frequency of several GHz. Although ferrite is well-known as a core material, it can only maintain an inductance below $10 \mathrm{MHz}$ [2, 9]. Core material made of nanoparticles should work in the high frequency regime. Thus, the magnetic property of the core may overcome the limitation of Sneak's low in the high frequency regime. Some studies on new core materials have already been conducted $[3,6]$. Research on the magnetic characteristics of small particles such as gold or platinum has being conducted [2, 10-11]. Metal nanoparticles are considered to have enhanced magnetics. In accordance with Hunt's law, a magnetic moment of small metal particles is strongly produced by electrons on the surface of the particles, which is what makes the particles magnetic [2]. Bulk Al is normally known to be non-magnetic. However, authors fabricated Al nanoparticles with a mean diameter of less than $20 \mathrm{~nm}$ from Al oxide (alumina) particles [12-16], and obtained Al nano-polycrystalline structures using the Al nanoparticles [15]. SQUID measurements of the magnetic hysteresis revealed that the Al nano-polycrystalline structure was ferromagnetic. Using this method, sintered Al nano-polycrystalline structures from $\mathrm{Al}$ oxide powder can be easily fabricated at low cost in a short time [12-17]. These nano-metals with small poly-crystalline structures are novel materials, and their magnetic and mechanical properties, aside 
from some of the electrical properties, have not yet been clarified. It is of academic interest to know the origin of the magnetics for $\mathrm{Al}$ nano-polycrystalline structures because this will help us develop new methods to enhance the magnetics of other magnetic materials. Their density is almost the same as that of Al bulk, which means that the weight of electrical devices can be reduced. Al nano-polycrystalline substances should be applicable to cored inductors working at low and high $(\mathrm{GHz})$ frequencies [7] and electromagnetic waves absorber [8]. In this study, authors fabricated a core inductor with a sintered $\mathrm{Al}$ nano-polycrystalline structure for the first time and investigated whether the structure works as a core of inductor working at high frequency.

\section{Experimental Setup}

Al nanoparticles were obtained by using laser ablation in liquids. Al nanoparticles with a mean diameter of $19 \mathrm{~nm}$ fabricated from Al oxide (alumina) particles (purity: 99.9\%, Koujyundo Co. Ltd., Japan) were produced from $\mathrm{Al}$ oxide particles with a few $\mu \mathrm{m}$ using laser ablation in liquids with a high-repetition pulse laser [15-16]. Reduction by laser ablation in liquids was conducted in pure water [15]. An Nd: YAG pulse laser with a lasing wavelength of $1064 \mathrm{~nm}$ and a receptivity of $20 \mathrm{kHz}$ was used for the laser ablation in liquid. Al nanopastes with reduced $\mathrm{Al}$ nanoparticles were synthesized. XRD analysis revealed that the mean diameter of the produced $\mathrm{Al}$ nanoparticles was $19 \mathrm{~nm}$ [15]. However, the $\mathrm{Al}$ particles are secondary particles. The mean diameter of Al nanoparticles should be $2 \mathrm{~nm}$ or less. Producing any kind of nanoparticle with a diameter close to $2 \mathrm{~nm}$ in liquids is easily possible. It has been proven in various papers in which scanning transmission electron microscope (STEM) analyses were performed, and the structures and sizes have already been observed [12-14]. The size of the produced nanoparticles is not changed by materials. Additionally, the proof of the mean size, i.e., the size of the produced Si nanoparticles needed for them to emit violet light, should be lower than $2 \mathrm{~nm}$, and the bandgap energy enhanced by the size effect must be larger than $2 \mathrm{eV}$ [12-14].

Al nano-polycrystalline substances were fabricated by sintering $\mathrm{Al}$ nanopastes with the reduced $\mathrm{Al}$ nanoparticles. $\mathrm{Al}$ nanopastes were pasted onto Machol ceramic (Corning) four times to construct a core inductor. The size of the ceramic was $14 \times 19 \mathrm{~mm}$ with a thickness of $0.5 \mathrm{~mm}$. The total thickness of the $\mathrm{Al}$ nanopastes was $0.3 \mathrm{~mm}$, and they were sintered four times by an electrical hot plate. A copper wire coil with a diameter of $0.4 \mathrm{~mm}$ was wound ten times around the center of the ceramic. The length of all coils was $7 \mathrm{~mm}$. Core inductors using these materials (called sheet samples) were constructed. Al nano-polycrystalline structures were obtained by sintering the $\mathrm{Al}$ nanopastes at 250 degrees with a hot plate. Additionally, glycerol glycerin with the weight of $100 \mathrm{mg}$ was mixed into the Al nanopaste before sintering to increase the volume resistivity. A rod-type Al nano-polycrystalline substance (called a rod sample) with the size of $4 \times 1 \times 8 \mathrm{~mm}^{3}$ was also fabricated. The thickness of the $\mathrm{Al}$ nano-polycrystalline substance with high volume resistivity sintered on the Machol plate was $0.5 \mathrm{~mm}$.
The generation of eddy currents owing to magnetization rotation on the hard axis of the Al nano-polycrystalline substance magnetization resulted in reduced inductance in the high frequency regime. To make core inductors work in the high frequency regime, we need to make the volume resistivity high enough to protect the eddy current.

The magnetic hysteresis of the reduced $\mathrm{Al}$ nanoparticles and the Al nano-polycrystalline substance was measured using a SQUID. The structure of the sintered Al nanopastes were observed by an scanning electron microscope (SEM) (S-4700, Hitachi High Technologies, Japan). An energy-dispersive X-ray spectrometry (EDX) (EMAX7000, Horiba, Japan) was used for analyzing the distributions of silver, mixed metal, oxides. An LCR meter (3532-50 LCR High Tester, Hioki, Japan) was used to measure the inductance of the inductor. Also, the inductance of a no-core inductor with Machol ceramic and a copper wire coil was measured for a comparison with the core inductor. Here, the inductance was measured from $42 \mathrm{~Hz}$ to $5 \mathrm{MHz}$. The Q-factor of the cored inductors was also measured.

\section{Results and Discussion}

Fabricated core inductors using sheet samples are shown in Figures 1 (a) and (b). Core inductors with low volume resistivity (metal) and high volume resistivity is shown, the structure is shown in Figure 1(c). A magnetic hysteresis curve of the Al nano-polycrystalline body with low volume resistivity at a temperature of $4.2 \mathrm{~K}$ measured by SQUID is shown in Figure 2. By measuring the magnetic hysteresis using a SQUID, it was found that the nano-polycrystalline structure was ferromagnetic. The synthesized Al nanoparticles were paramagnetic, which was judged by the measured magnetic hysteresis curve. Reduced Al nanoparticles cannot be attracted by magnets, and neither can sintered $\mathrm{Ag}$ nanopaste. These results lead us to conclude that the magnetic interaction among $\mathrm{Al}$ nanoparticles is enhanced because the distance between $\mathrm{Al}$ nanoparticles is very close. From $4.2 \mathrm{~K}$ to $300 \mathrm{~K}$, in the M-H curve, a clear hysteresis is evident, which demonstrates that the Al nano-polycrystalline substance is ferromagnetic. The coercive force of the $\mathrm{Al}$ nano-polycrystalline substance was estimated to be 300 Oe. The saturated magnetic field intensity was estimated to be 2000Oe. However, it has been considered that the cause that the sintered Al polycrystalline has ferromagnetism should not that the pure Al nanocrystals changed to having ferromagnetism. It has been considered that the cause will be 1) mixing inpurities to $\mathrm{Al}$ nanocrystals, such as oxygen, to the $\mathrm{Al}$ nanoparticles, and 2) lattice defects.

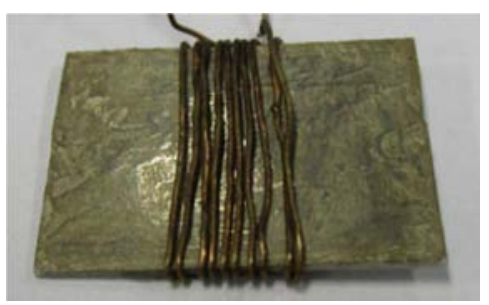

(a) 


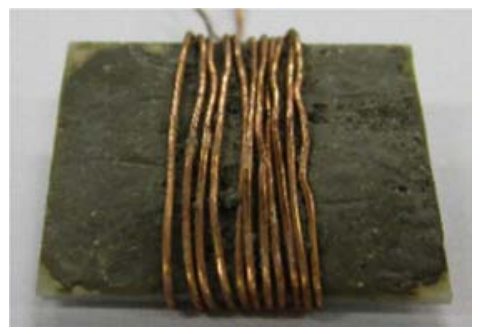

(b)

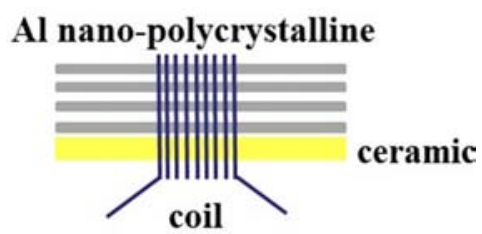

(c)

Figure 1. Fabricated cored inductor with Al nano-polycrystalline substance. (a) Low volume resistivity (metal), (b) High volume resistivity, (c) Structure (corresponding to Figure 1a).

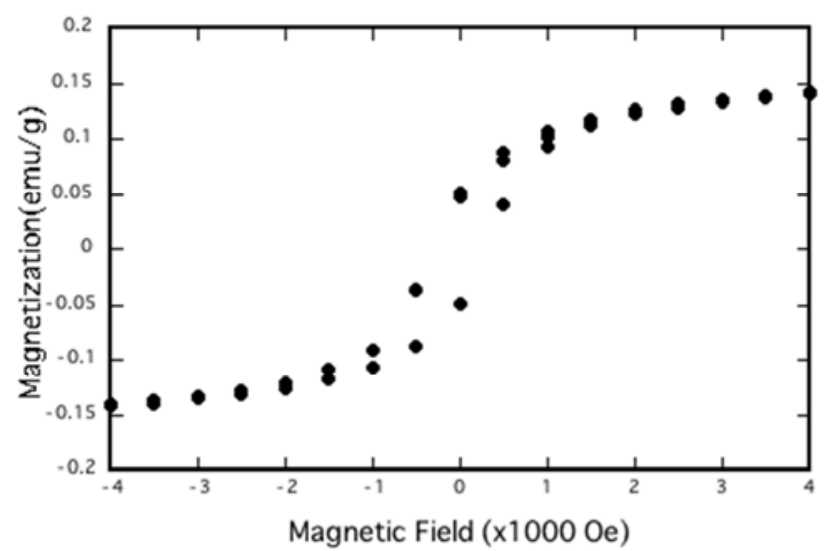

Figure 2. Magnetic hysteresis curve of the Al nano-polycrystalline body measured by SQUID.

The results of SEM analysis are also shown in Figure 3. Inductor cores with low volume resistivity (metal) and those with high volume resistivity are shown in Figures 3 (a) and 3 (b), respectively. These are secondary electron images. When using the sheet sample with low volume resistivity (metal), as shown in Figure 3 (a) it was found that the sintered $\mathrm{Al}$ nanopaste consists of $\mathrm{Al}$ particles with a diameter below $1 \mu \mathrm{m}$. The boundary line between the large particles can not be distinguished, although they are connected with each other. Secondary large Al particles with the mean diameter of less 1 $\mu \mathrm{m}$ exist in them, and $\mathrm{Al}$ nanoparticles with the mean diameter of a few nm are set tightly by self-organization in a single large $\mathrm{Al}$ particle. The nano-polycrystalline substance contained an impurity of $5 \% \mathrm{Ag}$ nanoparticles. The $\mathrm{Al}$ particles and Ag nanoparticles were separately separated. The evaluated volume resistivity was 3 times higher than that of conventional Al bulk. It had been found from the SEM image that nano-order small structures exist on the surface of the secondary aluminum particles.

Small Al nanoparticles with a diameter below $5 \mathrm{~nm}$ partially remained without being sintered. When sintering the
$\mathrm{Al}$ nanopaste, the mean size of the $\mathrm{Al}$ nanocrystals is maintained because the sample was sintered at a low temperature, and the large $\mathrm{Al}$ particles contain numerous $\mathrm{Al}$ nanoparticles in three dimensions.

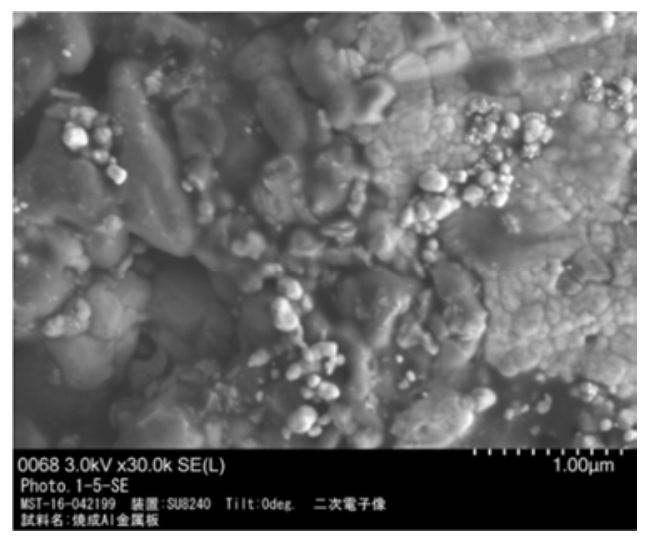

(a)

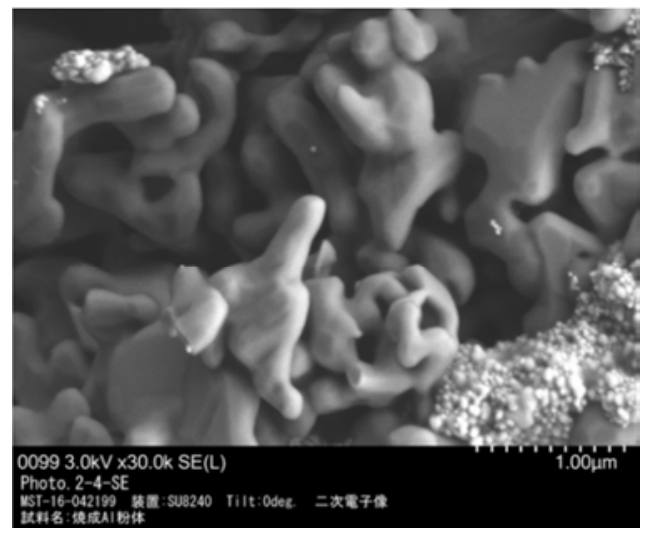

(b)

Figure 3. Results of SEM analysis. (a) Using core with low volume resistivity (metal), (b) Using core with high volume resistivity. The magnification of SEM image is both 30000 times.

When using the sheet sample with high volume resistivity, as shown in Figure 3 (b) it was found that numerous discontinuous sponge structures were constructed. The structures are not connected to each other. The volume resistivity of the fabricated sintered $\mathrm{Al}$ nanopaste was $10^{7}$ times $(200 \mathrm{k} \mathrm{ohm})$ higher than that of Al bulk. This is because many carbon and oxygen impurities exist between the $\mathrm{Al}$ particles with mean diameters below $1 \mu \mathrm{m}$.

Results of the characteristic X-ray spectrum of the sintered Al nanopastes are shown Figure 4. Cores with low volume resistivity (metal) and those with high volume resistivity are shown in Figure 4 (a) and 4 (b), respectively. According to the characteristic X-ray spectrum, silicon, iron and potassium were detected in the secondary $\mathrm{Al}$ particles. However, the ratio of all the impurity was in the order of $0.001 \%$ in the metal oxides before sintering. This impurity content is not a problem in regard to fabricating pure metals plates. The impurities exist mainly in the secondary aluminum particles. The EDX analysis showed that oxygen or carbon was attached to the outer surface of the secondary Al particles. 


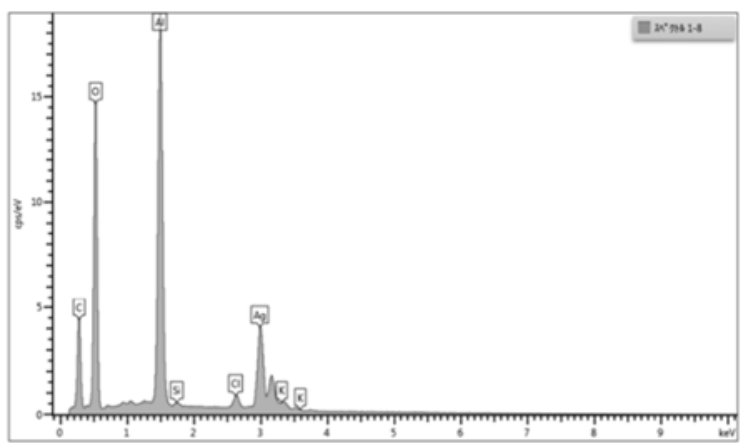

(a)

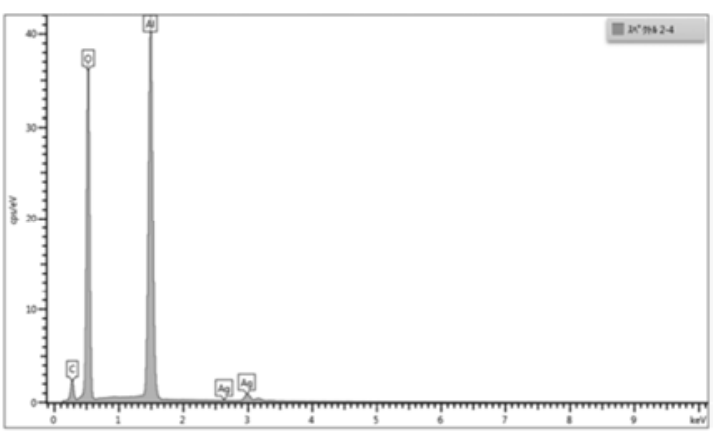

(b)

Figure 4. Measured characteristic X-ray spectrum of the sintered Al nanopastes. (a) Core with low volume resistivity (metal), (b) Core with high volume resistivity.

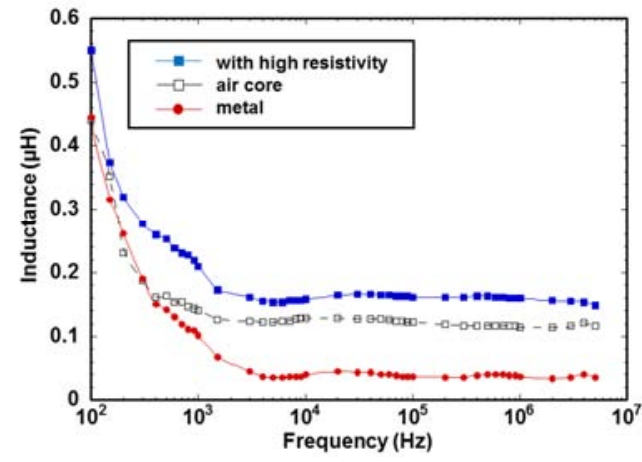

(a)

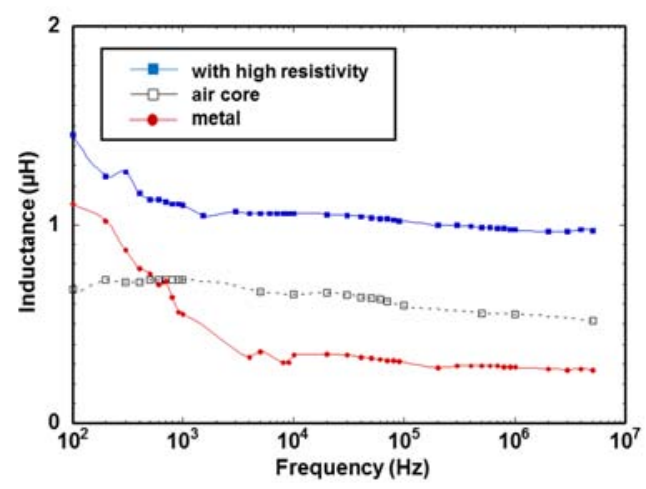

(b)

Figure 5. Measured inductance of Al nano-polycrystalline substance. (a) Rod sample, (b) Sheet sample.

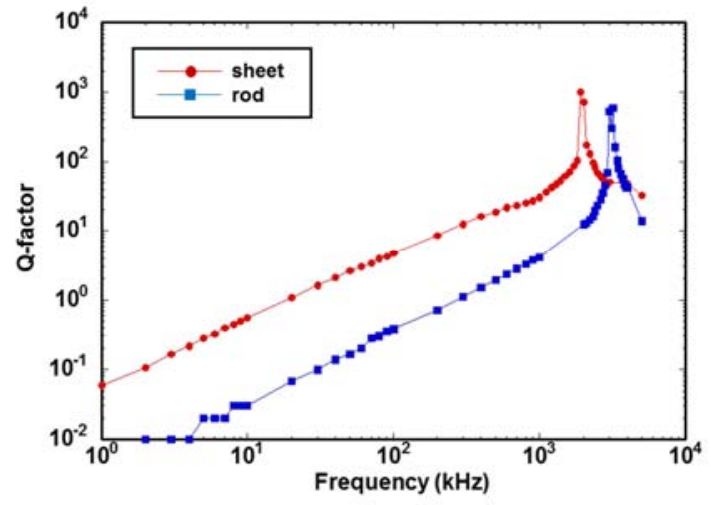

Figure 6. Evaluated Q-factor.

The dependencies of frequency on the inductance of the fabricated core inductors are shown in Figures 5(a) and 5(b). The measured inductance of the $\mathrm{Al}$ nano-polycrystalline substance with low volume resistivity was lower than the air cored inductor. This is because eddy currents are generated in the $\mathrm{Al}$ nano-polycrystalline substance and protect the generation of line of magnetic force. Measurement results of the inductance of the Al nano-polycrystalline substance with high volume resistivity showed a degradation from $100 \mathrm{~Hz}$ to $4 \mathrm{kHz}$. The inductance of $0.16 \mu \mathrm{H}$ for the $\mathrm{Al}$ nano-polycrystalline substance with high volume resistivity was kept from $4 \mathrm{kHz}$ to $5 \mathrm{MHz}$. This value is higher than that of the air core inductor.

Comparing the measured inductance of the $\mathrm{Al}$ nano-polycrystalline substance with high volume resistivity shown in Figures 5(a) and 5(b), it can be found that the inductance of the plate sample is higher than that of the rod sample. It seems this is because the plate sample had a larger resistance owing to its large size. The large resistance should result in reducing the eddy currents of the sample. As shown in Figure $5(\mathrm{~b})$, the inductance of $1 \mu \mathrm{H}$ for the $\mathrm{Al}$ nano-polycrystalline substance with high volume resistivity was kept from $4 \mathrm{kHz}$ to $5 \mathrm{MHz}$. For the air core inductor, the inductance degraded gradually as a function of frequency owing to capacitance between copper wires. From $100 \mathrm{~Hz}$ to 4 $\mathrm{kHz}$, a large degradation of inductance was evident, as shown in both Figures 5(a) and 5(b). These results indicate that $\mathrm{Al}$ nanoparticles in the Al nano-polycrystalline substance had some easy axes of magnetization as well as Iron. Magnetic resonance occurred, and inductance degraded radically at a low frequency. Evaluated Q-factors are shown in Figure 6. Both samples had high Q-factors of over 100 at the frequency of a few MHz. Next, the calculation for the relative permittivity of magnetic material used for the core $[2,18]$ is shown. The effective relative permittivity is shown as.

$$
\mu_{r 2}=\eta \cdot \mu_{r e f f}=\frac{\eta}{\frac{1}{1+\mu_{r e}}+N_{z}} .
$$

The demagnetization factor is shown as

$$
N_{z}=\frac{1}{n+1+\frac{n}{m}} \text {. }
$$


Thus, the next equation of relative permittivity from Eqs. (1) and (2) is obtained as

$$
\mu_{r}=\frac{1}{\frac{1}{\mu_{\text {rexp } / \eta}}-N_{z}}-1
$$

For the rod sample, $\mathrm{n}$ and $\mathrm{m}$ were set to be 4 and 8 , respectively. The $N_{z}$ calculated by Eq. (2) was $1 / 11$. For the sheet sample, $m$ and $n$ were set to be 38 and 26, and the $N_{z}$ calculated by Eq. (2) was $1 / 40$. Here, $\eta$ is the ratio of core area to coil area; for both samples, this was set to 0.33 . The calculated results of the initial relative permittivity by using Eq. (3) and the data of the inductances for $\mathrm{Al}$ nano-polycrystalline substance with high volume resistivity are shown in Figure 7, where relative permittivity was obtained by calculations from the ratio of the inductance of the core inductor with Al nano-polycrystalline substance to the one of the air core inductor.

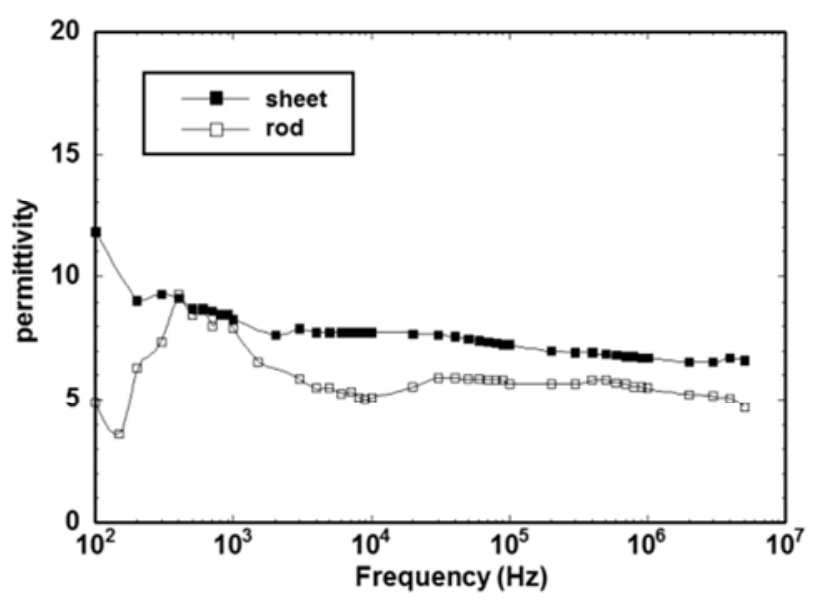

Figure 7. Evaluated relative permittivity.

Here, to disregard the degradation of inductance due to capacitance between copper wires at a high frequency, the inductance of the air core inductor at $50 \mathrm{kHz}$ was used to calculate the relative permittivity of the core material. The effect on the inductances from increasing capacitance between copper wires owing to the Machol ceramic was negligible. It was found that the evaluated relative permittivity of the $\mathrm{Al}$ nano-polycrystalline substance was 7 at the frequency of 1 $\mathrm{MHz}$. The relative permittivity was lower than that of the $\mathrm{Fe}$ particles. Using Fe particles with a mean diameter of $\mu \mathrm{m}$ as core material, almost the same relative permittivity was obtained $[2,3]$. The calculated relative permittivity of the rod sample was smaller than that of the sheet sample because of the low resistance at a frequency of over $2 \mathrm{kHz}$. For both the sheet and the rod samples, no large degradation of relative permittivity in the few $\mathrm{MHz}$ regime owing to magnetic resonance was found. This is because the mean size of the $\mathrm{Al}$ nanoparticles was below $2 \mathrm{~nm}$. However, the relative permittivities were gradually degraded. The equation for calculating the magnetic resonance frequency is shown as [2].

$$
f_{r}=\frac{\gamma}{2 \pi}\left(H_{k}\right)
$$

where $\gamma$ is the rotation magnetic coefficient, $\mathrm{H}_{\mathrm{k}}$ is the saturated magnetic field intensity (set to $2000 \mathrm{Oe}$ ), and where the magnetic resonance frequency was calculated to be $5.6 \mathrm{GHz}$. Thus, it is expected that these inductors to work at a frequency of a few $\mathrm{GHz}$ if the $\mathrm{Al}$ nano-polycrystalline substance has high resistivity and the mean size of its comprised $\mathrm{Al}$ nanoparticles is adequately small. It is because 1) smaller metal nanoparticles have enhanced magnetics (explained at chapter 1 in the reference [2]), and core inductors with high inductances can be obtained. Also, 2) the generation of the eddy current should be reduced at a high frequency. The working frequency will mainly be decided by the resonance frequency and the eddy currents. The generation of the eddy current will be degraded at a high frequency owing to the increased volume resistivity. Ratio of surface to volume for the secondary produced particles increases, and spaces will increase. Also, when a material consists of metal nanocrystals with small diameter, electron scattering occurs remarkably at the surface of the metal nanocrystals. They result in increasing the volume resistivity. The results of the SEM analysis in the text evidently show the existence of very small $\mathrm{Al}$ nanoparticles with a size below $5 \mathrm{~nm}$.

\section{Conclusions}

Authors showed that the aluminum nano-polycrystalline body is ferromagnetic by measuring the magnetic hysteresis curve using a SQUID. Also, an inductor core using an $\mathrm{Al}$ nano-polycrystalline substance was fabricated for the first time. Inductances of the core inductor with frequency dependence were measured. Results showed that the core inductor using Al Nano-polycrystalline substance with high volume resistivity works at the high frequency of $5 \mathrm{MHz}$. The relative permittivity of the Al nano-polycrystalline substance with high volume resistivity evaluated by numerical analysis was 7 at a frequency of $1 \mathrm{MHz}$. The magnetic resonance frequency of the Al nano-polycrystalline substances were evaluated to be $5.6 \mathrm{GHz}$. This inductor will work at a frequency of a few $\mathrm{GHz}$.

\section{Acknowledgments}

This study is partially supported by MEXT-Japan for the Strategic Research Foundation at Private Universities, under Grant No. S1511031.

\section{References}

[1] J. I. Martın, J. Nogues, K. Liu, J. L. Vicent, I. K. Schuller, "Ordered magnetic nanostructures: fabrication and properties", J. of Magnetism and Magnetic Materials, 2003, 256, (1), pp. 449-501.

[2] Function Investigation Committee of Nanoscale Magnetic Material.: "Nanostructured magnetic materials -physics, function, design-", (Kyoritsu Publishing, Tokyo, JAPAN, 2010), Chapter 1 and 2. 
[3] Y. Shimada, M. Yamaguti, S. Okamoto, O. Kitakami, G. W. Qin K. Oikawa, "Enhanced initial permeability of composite assembly of ferromagnetic particles", J. Magn. Soc. Jpn., 2006, 30, pp.540-544 [in Japanese].

[4] R. Tang, M. Mizuguchi, H. Wang, R. Yu, K. Takanashi, "Strong temperature dependence of magnetoresistance in Co-C granular thin films", IEEE Trans. Magn., 2010, 46, pp.2144-2147.

[5] S. Yoshida, S. Ando, Y. Shimada, K. Suzuki, K. Nomura, K. Fukamichi, "Crystal structure and microwave permeability of very thin $\mathrm{Fe}-\mathrm{Si}-\mathrm{Al}$ flakes produced by microforging”, J. Appl. Phys., 2003, 93, pp.6659-6661.

[6] V. Korenivski, "GHz magnetic film inductors", J. of Magnetism and Magnetic Materials, 2000, 215-216, pp.800-806.

[7] Y. Gao, S. Z. Zardareh, X. Yang, T. X. Nan, Z. Y. Zhou, M. Onabajo, M. Liu, A. Aronow, K. Mahalingam, B. M. Howe, G. J. Brown, and N. X. Sun, "Significantly Enhanced Inductance and Quality Factor of $\mathrm{GHz}$ Integrated Magnetic SolenoidInductors With FeGaB/A12O3 Multilayer Films", IEEE Trans. on Electron Devices, 2014, 61(5), pp.1470-1475.

[8] P. Marín, D. Cortina, and A. Hernando, "Electromagnetic Wave Absorbing Material Based on Magnetic Microwires", IEEE Trans. on Magnetics, 2008, 44(11), pp.3934-3937.

[9] G. Howatt, R. Breckenridge,J. Brownlow, "Fabrication of Thin Ceramic Sheets for Capacitors", J. Am. Ceram. Soc., 1947, 30, pp.237-242.

[10] G. Herzer, "Grain structure and magnetism of nanocrystalline ferromagnets", IEEE Transactions on Magnetics, 1989, 25, (5). pp. 3327-3329.

[11] Lou, L., Hou, F. C., Wang, Y., Li, H. L., Li, W., Guo, D. F., Li,
X. H. and Zhang, X. Y.: 'Texturing for bulk $\alpha-\mathrm{Fe} / \mathrm{Nd} 2 \mathrm{Fe} 14 \mathrm{~B}$ nanocomposites with enhanced magnetic properties', J. Magn. Magn. Mater., 2014, 352, pp.45-48.

[12] K. Saitow and T. Yamamura, "Effective Cooling Generates Efficient Emission: Blue, Green, and Red Light-Emitting Si Nanocrystals", J. Phys. Chem. C, 2009,113(19), pp.8465-8470.

[13] V. Švrček, T. Sasaki, Y. Shimizu, and N. Koshizaki, "Blue Luminescent Silicon Nanocrystals Prepared by Ns Pulsed Laser Ablation in Water", Appl. Phys. Lett., 2006, 89, 213113-1-3.

[14] I. Umezu, A. Sugimura, M. Inada, T. Makino, K. Matsumoto, and M. Takata, "Formation of Nanoscale Fine-Structured Silicon by Pulsed Laser Ablation in Hydrogen Background Gas", Phys. Rev. B, 2007, 76, 045328-1-10.

[15] T. Saiki, T. Okada, K. Nakamura, T. Karita, Y. Nishikawa, and Y. Iida, "Air Cells Using Negative Metal Electrodes Fabricated by Sintering Pastes with Base Metal Nanoparticles for Efficient Utilization of Solar Energy", Int. J. of Energy Science, 2012, 2(6), pp. 228-234.

[16] T. Saiki, Y. Iida, K. Ri, M. Yoshida,Y. Koga, "Electrical property of laser-sintered nanopastes with reduced metal nanoparticles prepared by laser ablation in liquids", $A d v$. Materials, 2014, 3(6), pp.75-88.

[17] T. Saiki and Y. Iida, "Fabrication of Sintered Si Nano-polycrystalline with Reduced $\mathrm{Si}$ Nanoparticles and Property of Photoluminescence in Visible Regime for Sintered Si Nano-polycrystalline by Violet Light Excitation", American Journal of Nano Research and Applications, 2015, 3(5), pp.82-88.

[18] M. Sato, and Y. Ishii, "Simple and approximate expressions of demagnetizing factors of uniformly magnetized rectangular rod and cylinder", J. Appl. Phys., 1989, 66, pp.983-98. 